\title{
Sport integrity systems
}

\section{A recommended system for promoting and safeguarding sport integrity}

\author{
Lisa A. Kihl
}

\section{Introduction}

Globally, National Sport Governing Bodies (NSGBs), for example in Australia, Czech Republic, United Kingdom and United States of America, struggle to prevent and address integrity issues is not a new phenomenon (Kihl 2019). For example, during the past five years the Australian National Rugby League has experienced numerous on and off-the-field integrity issues including positive doping cases, salary cap breaches, player disorderly conduct, domestic assault, and sexual misconduct by different actors in several of their clubs, e.g. Cronulla Sharks, Canterbury Bankstown Bulldogs, Melbourne Storm, Parramatta Eels (Kinsella 2019). For over 20 years, Larry Nassar was enabled to sexually abuse more than 300 female athletes. Poor governance, ineffective oversight, and cover-ups by the Unites States Olympic and Paralympic Committee, USA gymnastics, and Michigan State University all contributed to Nassar's abuse. Whilst in 2019, UK athletics was labelled "the most dysfunctional Olympic sport in Britain" following a series of issues related to questionable leadership and relationships, doping, and substandard safeguard procedures (Ingle 2019, p. 6). The range of integrity violations illustrate the global need for NSGBs to rethink how they promote integrity and minimise integrity risks and violations by way of good governance and independent policy reforms.

The broad nature of sport integrity requires a multi-lens systems approach to promoting integrity and limiting risks and violations. Integrity risks are "conditions and behaviours within an organisation that increase its vulnerability to the occurrence of integrity violations" (Molina 2018, p. 873).

Integrity violations include both unethical legal behaviours and activities (e.g. abuse of power, code of conduct breaches conflict of interest, gamesmanship, poor governance practices, sport code violations) and unethical illegal behaviours (e.g. bribery of officials, illegal sport betting, match fixing, money laundering). A sport integrity system consists of individuals, institutions, policies, practices, and agencies that contribute to fostering and safeguarding the integrity of an NSGB (Kihl 2019). The central focus of an integrity system is outlining the components and conditions necessary for preventing integrity violations and limiting integrity risks in governance and sport competitions. 
This chapter will briefly review sport scholarship on what is known about the effectiveness of good governance and policy instruments for addressing integrity violations and fostering integrity. The review leads to the conclusion that promoting and safeguarding integrity requires a system that includes sport actors, accountability mechanisms, and integrity management. Next, the different components that comprise a sport integrity system and their interactions are outlined. The chapter concludes with contribution of designing a sport integrity system and suggestions for future research.

\section{Description of current practice and the problems that this entails}

The strategy of choice for academics and sport practitioners for restoring integrity, in general, is to focus on a "Band-Aid response to particular emergencies" (e.g. such as doping, bribery, match fixing, sexual assault)" by adopting individual policies rather than promoting and safeguarding integrity through a coordinated holistic system (Cleret et al 2015; Gardiner et al. 2017, p. 16). As such, sport scholars and experts have leaned on two main reactive strategies to curb specific integrity violations: (1) implementing good governance principles (e.g. Chappelet 2018; Chappelet \& Mrkonjic 2013; Geeraert, Alm \& Groll 2014; Henry \& Lee 2004), and (2) developing and implementing different individual policies (e.g. anti-doping, manipulation of sport, Safe Sport, concussion, gender equity, anti-racism). After a series of scandals (e.g. corruption and sexual assault) in both national and international sport organisations there was a call for good governance and extensive contributions to scholarship have been made. The literature has drawn from cooperate governance and democratic governance theory, where a series of national sport good governance publications (e.g. Chappelet \& Mrkonjic 2013; Geeraert 2018; Henry \& Lee 2004) have put forth a variety of principles to promote good governance and fight against integrity breaches (Chappelet 2018; Di Marco 2019). In the sport governance literature, integrity is perceived as a principle of good governance rather than a quality of sport and/or sport system. For example, Chappelet and Mrkonjic's (2013) Basic indicators for better governance in international sport lists integrity as a principle to adhere to in seeking good sport governance. Numerous countries have implemented different principles of good governance in their respective NSGBs (e.g. Australia, Belgium, Brazil, Canada, Denmark, Japan, Norway, and the UK). Despite the adoption of these principles, we continue to see integrity risks and violations.

In this chapter, I am arguing that sport governance is an internal element of an overarching sport integrity system. An integrity system is much broader and involves both internal and external mechanisms and actors that aim for high integrity of NSGBs. Good governance in sport governing bodies is argued to be "the means to the end" in gaining organisational "legitimacy, effectiveness, and resistance to unethical practices" (Geeraert 2018, p. 7). However, its precise 
meaning is contested and the application of the long list of indicators seems daunting.

The second approach used to curb corruption is the ratification of independent policies (Kihl 2019). As different types of rorting, sexual assault, and harassment cases have surfaced, NSGBs have tended to adopt separate policy instruments to counteract these unethical and/or illegal activities. For example, anti-doping, anti-harassment, betting and crime reporting, equity and diversity, match fixing, and Safe Sport are the prevalent policies of many NSGBs. Depending on the country, the sport, and the integrity issues that are most pressing influence the policies that are implemented. An emerging trend is NSGBs with the financial capacity is hiring integrity officers to oversee the implementation of specific policies. For example, USA NSGBs require a safe sport program and an integrity officer whose role is oversee the prevention of sexual assault and harassment. Recurrent integrity violations within NSGBs strongly suggest that the various policy mechanisms adopted to control violations are inadequate and detached to respond to the variety and range of integrity risks and breaches they encounter (Kihl 2019).

For example, Australia and Finland have adopted specific sport integrity initiatives. Sport Integrity Australia is responsible for 'Safeguarding the Integrity of Sport' with a focus on policy development, intelligence, investigations, education and training to counter doping, match manipulations, child safety, and protection from harassment. The Finish Center for Integrity in Sports focuses on advocating ethical principles in sport that focus on anti-doping, prevention of match-fixing, spectator violence, and fair play. Whilst these sport integrity initiatives are extremely important and a good first step in safeguarding integrity, arguably these programs do not reflect a holistic integrity system. Furthermore, there is an emphasis on safeguarding integrity from certain unethical activities rather than acknowledging the multifaceted nature of sport integrity which requires a system approach that both safeguards and promotes integrity. By not acknowledging the multifaceted nature of integrity leads to safeguarding prioritised unethical behaviours whilst other types of unethical behaviour go unchecked and can prosper.

Sampford, Smith and Brown (2005, p. 100) suggested "institutions and relationships, are often weak individually but collectively potentially strong”. Arguably the lack of clarity and understanding of the concept of sport integrity (Cleret et al 2015; Gardiner et al. 2017), along with the reactive governance in applying band aid and piecemeal reforms that aim to safeguard integrity requires a radical shift in how NSGBs go about doing integrity. To curb the persistent integrity risks and violations and promote integrity in NSGBs, requires more than good governance, integrity officers, and specific integrity policies and units. Institutionalising and sustaining integrity in NSGBs is contingent on a holistic system comprised of interdependent and cooperative actors, an internal integrity management infrastructure, and different internal and external accountability mechanisms (Kihl 2019; Sampford et al. 2005). 


\section{Description of an alternative approach: Sport integrity system}

NSGBs are complex organisations (Pedras, Taylor \& Frawley 2020) that encounter a myriad of integrity concerns. These national governing bodies are multi-levelled and endure external and internal environmental influences that generate a variety of novel and reoccurring integrity risks and violations commanding thoughtful management reflection. For example, the current COVID-19 crisis has created unique economic conditions, cultural influences, and political pressures on organisations regarding athlete well-being, training, and return to play. COVID-19 crisis and the related economic crisis has made NSGBs particularly vulnerable to different form of corruption including match fixing (in particular Ghost games), money laundering, and transnational crime (Kihl \& Ordway forthcoming). Navigating novel and reoccurring integrity risks and violations requires more than just good governance. Integrity system theory posits a holistic perspective that focuses on the bigger picture and considers all the components and conditions necessary to the integrity of a sport organisation (Six \& Lawton 2013). It "focuses on the connection between various components within and outside the organisation, how they are interconnected and how they are jointly responsible for the integrity performance of an organisation" (Huberts \& Van Montfort 2020, p. 454). All components are interrelated, interdependent, and serve as the building blocks to a national integrity system (Pope 2008). A national sport integrity system includes actors who are responsible for coordinating and implementing the system, an integrity management infrastructure, and different accountability mechanisms (Huberts \& Van Montfort 2020).

A sport integrity system is based on the notion that there is no one solution to preventing unethical behaviours; rather, it requires "institutionalisation of integrity" by several agencies, actors, laws, regulations, practices, and ethical policies (Head 2012, p. 9). For example the International Federation of Association Football (FIFA) member associations' integrity programs possess the independence to create and implement their own program. Recognising each countries' football federation is distinctive in terms of its governance system, economics, history, and culture, social development; thus allowing each confederation the autonomy to design and implement an integrity system based on that it corresponds with their respective strategic priorities (FIFA 2020, par. 2). As such, NGSBs have the flexibility to determine which actors, the type of integrity management infrastructure, and the appropriate accountability mechanisms in designing their integrity system.

The internal mechanism of an overall integrity system is an integrity management infrastructure (also referred to as ethics management), which is its internal operational arm. Ashby's (1991) principle of requisite variety argues that the complexity of a system dictates the variety of monitoring and controlling systems to promoting and safeguarding integrity. An integrity management infrastructure has four functions: (a) determining and defining integrity, (b) guiding towards integrity, (c) monitoring integrity, and (d) enforcing integrity (Maesschalck \& Vanden Auweele 2010). Assessing relevant integrity actors and other stakeholders' 
perceptions of integrity and what values they aim to espouse in decision making, policy making, procedures, and processes is an important first task. This definition of integrity moulds the overall integrity culture through its mission and vision, rituals, symbols, structure, leadership and daily practices. Core integrity structures are devised and instruments selected that help promote, monitor, and enforce integrity. These include a combination of good governance principles (e.g. values-based mechanisms, compliance based mechanisms, structural, environment, equity, and political orientated structures and instruments).

Values-based mechanisms and compliance-based mechanisms are the two most commonly adopted ethical management orientations used to limit integrity risks and violations in sport governance. Values-based mechanisms (e.g. ethical codes, values statements, leadership) promote integrity and limit risks by using individual intrinsic motivation techniques that's build moral competence and integrity. In combination these techniques build an ethical culture that promotes ethical dialogue about integrity concerns, promotion of ethical behaviour, and decision making. The promotion of ethical dialogue is critical to high integrity management because it is a proactive way to promote integrity and not be reactive to unethical behaviour and thus is a demarcation from good governance. Compliance-based mechanisms use formal external control methods to reduce risks and violations. Compliance systems commonly entail four formal systems: (a) communications of organisational ethical values and principles (e.g. ethics committees, mission statements, education and training programs) (Constandt, De Waegeneer \& Willem 2019; De Waegeneer, Van De Sompele \& Willem 2016); (b) surveillance (e.g. whistleblower program, an Ombudsman) (Warren, Gaspar $\&$ Laufer 2014); (c) sanctions and investigations that reward ethical behaviours and punish violators (Maesschalck \& Vanden Auweele 2010) and (d) formal organisational structures (e.g. authority structures, division of labour, good governance principles, and level of empowerment) (Treviño \& Nelson 2017). In addition, environmental practices that adhere to societal expectations regarding sustainability and global warming, social equity and political responsibility are all key integrity management orientations that may be implemented.

Integrity performance relies on effective internal coherence with instruments, structures, and processes all managed as one unit and interconnected across the entire NSGB integrity system. Auditing existing core integrity structures and instruments is necessary to determine if additional mechanisms need to be added to strengthened integrity promotion and safeguarding from risks (e.g. athlete wellbeing, racial harassment, financial internal controls). Reinforcing existing instruments and/or implementing additional ones is critical for system coherence and effectiveness. A core and vital part of integrity management strategy is instituting the correct and quality processes to assure the different instruments are rightly implemented. Whilst time consuming, regular integrity management evaluations are necessary to appropriately address risks but also proactively address internal and external environmental forces that may create novel integrity concerns. Consequently, the integrity management infrastructure will require modifications 
(e.g. revising policy documents), changes (e.g. how programs are delivered, monitoring practices, and intelligence sharing), and/or in some cases mending (e.g. enhancing the ethical leadership of board members or presidents, mending trust with external watch dog agencies and whistleblowers (Hoekstra \& Kaptein 2020).

\section{Importance of different forms of accountability}

The historical autonomy of sport organisations and persistence of integrity issues across NSGBs illustrates their inability to suitably self-regulate and/or generate a culture of integrity (Chappelet 2016, 2018; Geeraert 2019). Manoli, Comille and Downward's (2020) research on individuals' perceptions of sport integrity supports this argument. Individuals' believed that sport was unable to manage its integrity and was unmotivated to protect its integrity because of peoples continued engagement in sport. Good governance principles rely on free and fair elections and watchdog groups as the primary means of accountability. The complexity of NSGBs (Pedras et al. 2020) and the breadth and depth of integrity concerns they are confronted with, warrants an integrity system that is dependent on several integrated accountability mechanisms to manage and regulate unethical behaviour (Heinrich \& Brown 2017).

Integrity systems require four types of accountability: vertical accountability, horizontal accountability, mutual accountability, and social accountability (Heinrich \& Brown 2017). Vertical accountability is similar to the democratic principle of holding regular, free, fair and transparent elections (Di Marco 2019). Vertical accountability alone is deemed insufficient for accountability. Pope (2008, p. 19) argued officials escape reelection by an unsatisfied society "through a combination of secrecy (so that the electors are unaware of what is transpiring) and the building of systems of patronage" as well as "indulging in shortterm populist acts, which may be to the longer-term detriment of the public". Horizontal accountability represents the separation of powers and involves formal institutions who hold the authority to perform checks and balances (Howe \& Haigh 2016). Integrity institutions (i.e. organisations that have the responsibility of integrity) function to oversee the integrity of governing bodies, administrative units, and other institutions responsible for integrity operations. There are core integrity institutions (e.g. watchdog groups, integrity agencies, law enforcement) whose primary role is monitoring. Distributed integrity institutions play an auxiliary role of integrity monitoring in addition to their regular core business (e.g. governing, statutory bodies). As previously noted, good governance advocates tend to focus on vertical accountability and horizontal accountability.

Vertical and horizontal accountability do not sufficiently address the concern of 'who guards the guardians' (Howe \& Haigh 2016). Mutual accountability is a means for guarding the guardians through a collective set of institutions hold each other accountable by means of cross checking, coordination, consistency, and operational arm relationships (Sampford et al. 2005). Jurisdictions are defined and relationships are required between a president, governing board, integrity units, and ethics 
committees to ensure who is performing specific check and balance roles. Effective integrity management entails coordination and consistency of sport integrity policies. Actors carry out these integrity operations by implementing respective sport policy processes. Integrity performance entails a system of functioning networks where each actor is accountable to one or more actors (Mulgan 2003). Managing the various complex integrity relationships is critical in order to nurture relationships rather than creating barriers that can lead to non-cooperation.

Sport integrity systems also require civil society's buy-in to performing as an accountability arm. Societal accountability depends on the civic engagement of different actors to use their collective voice to hold NSGB actors accountable (Jayal 2008). Civil society actors involve private sector actors (e.g. media, sponsors), fans, and collective social movements to use their combined power to expose transgressions and place pressure on NSGBs to operate effective integrity systems. "To be effective, they have to generate sufficient public pressure to strengthen horizontal accountability and to create a credible threat of sanctioning power through the mechanism of electoral accountability" (Schatz 2013, p. 171). To date, civil society is not shown a consistent commitment to holding NSGBs accountable. Fans, sponsors, media, and the general public might display outrage in cases of severe unethical behaviour (e.g. athlete acts of sexual violence, administrative abuses of power and fraud); however, they do not hold the NSGB accountable and people continue to engage (Manoli et al. 2020). Good governance generally includes vertical accountability and strong internal accountability and control (similar to horizontal accountability). Internal accountability and control do not include external accountability institutions (i.e. integrity institutions). Furthermore, good governance does not include mutual or social accountability. The strength of a NSGB's integrity system and separates it from good governance is the interplay of vertical, horizontal, mutual, and social accountability mechanisms (Schatz 2013).

\section{Application to NSGBs: A sport integrity system}

An NSGBs integrity system comprises of three main components: actors, an internal integrity management infrastructure (i.e. operational arm), as well as external accountability guardians of the NSGBs administration and governing board. Components of the NSGBs integrity system and their respective relationships require coherent management to ensure integrity risk containment, including appropriate exercises of power. In such a system, depending on the NSGB's governance type (i.e. federated or unitary), NSGBs collaboratively support and coordinate with their respective sport network to implement specific measures, policies, and practices considered important to ensuring integrity. An NSGB also coordinates with the external accountability guards to assure cohesion with the internal environment and integrity actors. A sport integrity system is therefore multidimensional and elements comprising the system can vary significantly depending on the sport governing body, the level in which the elements operate, the location (e.g. urban or rural), and the political and economic environments. 


\section{Actors}

Several NSGB internal and external actors are responsible for generating and guarding integrity. Internal integrity actors oversee and coordinate the integrity management infrastructure as well as harmonising with external actors and institutions of the larger integrity system. The governance structure of the organisation (i.e. federated or unitary) will influence how the system is managed, coordinated, and the nature of accountability. The delineation of their respective roles and responsibilities is critical in coordinating initiatives at the local, regional and/or state levels accord with the overall national system. Furthermore, they are responsible for overseeing and managing the different actors administering the integrity management infrastructure across the system.

External actors (e.g. community, government actors, legal actors, media, sponsors and their integrity units, sport betting intelligence agencies) hold horizontal and social accountability roles. In the contexts of event management, the public sector (e.g. betting monitoring agencies, sponsors, broadcast agencies) will also take on mutual accountability roles in the implementation and communication of their respective security infrastructures. It is important that NSGBs identify actors who are internal and external to the system, as well as define their responsibility and roles at the club, regional, state, and national levels. The effective coordination of the various integrity actors within a national system is imperative.

\section{Internal environment}

The internal environment includes an integrity management infrastructure that is harmonised with the broader national integrity system. An integrity management infrastructure makes-up the internal environment and contains three main components: instruments, structures and processes. The goal of the integrity infrastructure is to institutionalise integrity throughout the NSGB byway of official and explicit guidelines that infuse ethical values into day-to-day sporting decisions and practices. The management infrastructure is custom built so as to parallel the NSGB's expectations for promoting and safeguarding their integrity vision. NSGBs are complex organisations that experience a variety of integrity concerns in the governance and delivery of sport; therefore, the integrity infrastructure will require an array of practices within each component.

\section{External environment}

The external environment contains guardians that function as external checks and balances. Checks and balances are the mechanisms for guardianship and accountability that ensure that local, regional, and national sport governing boards operate within legal and social boundaries. External guardians consist of an independent regulatory environment and the social environment that serve mutual, horizontal and social accountability roles. 
Regulatory environment refers to the laws, regulations, and external regulatory oversight agencies that serve to work in coordination with a sport integrity system. Standard regulatory watchdogs include law enforcement, state and national legislation, government regulations, and specific sport and non-sport regulatory oversight bodies including anti-corruption and safe sport agencies. These external regulatory agencies play mutual and horizontal accountability functions. For example, international anti-corruption agencies include the world anti-doping agency, INTERPOL, and government agencies such as the Australian National Integrity in Sport Unit who can aid NSGBs in combating corruption in sport. Safe sport agencies like the US Center for SafeSport aim to end different forms of abuse in sport. Integrity-specific watchdog groups have also emerged including organisations such as the Sport Integrity Global Alliance and the Sport Integrity Unit, which focus on promoting integrity by providing governance resources and leadership.

The social environment (e.g. media, the private sector, and community members) serves horizontal, social, and/or mutual accountability roles. In a sport integrity system, private sector agencies generally involve sponsors and sport betting monitoring. During sporting events sponsors might play a variety of integrity roles including security (e.g. Visa provides secure payment infrastructure), digital intelligence (e.g. Samsung). Intelligence is also shared by sport data companies (e.g. sport radar) who monitor betting patterns. The main role of the media and the community is to be active participants in ensuring NGSB transparency and accountability by raising concerns and demanding strong integrity systems.

By accessing public information, the media, in particular, play a critical role in social accountability by exposing and calling for acceptable accountability for integrity risks and violations. In particular, the media's reporting on corrupt practices such as illegal gambling on sport, match fixing, state doping systems, and poor governance practices (e.g. the USOC failure to address numerous cases of sexual harassment coaches and/or support staff) have assisted in exposing both systematic integrity risks and violations. Equally important is the persistent (a) reporting on the lesser so called 'scandalous' behaviours and activities not just the cases that make good headlines, and (b) scrutiny of NSGBs in that they suitably respond to 'fixing' the risks and insisting on accountability of individual and organisational offenders. Exposing both corrupt and unethical/legal integrity violations and cases of a lack accountability to the public pressures NSGBs and their partners to be suitably responsive to all integrity breaches and not just the sensational corruption cases.

\section{Conditions for effective functioning of components}

Ideally, an integrity system promotes integrity and minimises risks by creating functional systems, practices, and ethical decision making. An effective system requires sufficient capacity (e.g. resources, financial support, individuals) to support the functioning of the system, and involve coherent institutional cooperation 
(functional system interactions). Within and across the system, a NSGB ought to facilitate necessary human and financial resources to help realise their integrity goals. This requires coordination with local clubs and regional and state/provincial governing bodies to ensure polices and processes are suitably implemented.

Recognising the potential differences in the structure of NSGB and their sport integrity systems, the number and nature of institutions, policies, practices, actors, and agencies that make up the system is not prescribed. The country and nature (e.g. sport, funding, size, actors, location, and so forth) of the NSGB will influence which components are the best fit for an effective integrity system. A NSGB could quite possibly select a number of different configurations to achieve high integrity. Relevant NSGB stakeholders will need to determine the appropriate configuration of policy, practices and agencies that best suit their mission and context. Additionally, given the dynamic context that where sport operates and is delivered, the integrity system configuration requires adaptation over time. As new integrity risks emerge and integrity breaches occur, the system will require some adjustments to address such situations.

\section{Conclusion and implications for research}

In this chapter, I have argued that the NSGBs current approach to promoting and safeguarding integrity is not working. Scholars and experts perceive the solution to the persistence of integrity violations is the adoption of good governance principles and independent policies to ensure competitions are played honestly, athletes are protected, and governing boards engage in ethical behaviours and practices. Policy instruments and good governance principles are part of a whole integrity system. A sport integrity system entails actors, constructing an internal (integrity management infrastructure), and external (regulatory and social) environment, and using a range of accountability mechanisms. The complexity of NGSBs and the range of integrity concerns (new and previous) calls for integrity management to be comprised of different orientations. The effective functioning of components that make up a sport integrity system requires coordination, capacity building, and ongoing evaluation of the effectiveness of the system as a whole as well as its individual components. Individual NSGBs will need to determine how a sport integrity system is initiated and managed as well as decide the criteria for assessing high-performing integrity systems. Providing sufficient capacity support for local, regional, and state levels is essential to minimise system gaps that undermine overall performance. Last, to promote integrity and limit risks and violations NSGBs will need to develop performance targets and monitoring systems to evaluate their progress. Carrying out such performance appraisals can help build public trust by demonstrating that NSGB leaders are serious about integrity, and sport is perceived as being clean, fair, and justly managed.

The proposed sport integrity system provides several opportunities for future research. Gaining an understanding of different NSGBs integrity system configurations and how country and sporting culture may influence integrity 
management, accountability mechanisms and actors is a plausible first step. Empirical research that tests the proposed system, and thereby determining necessary adjustments is also an important theoretical contribution. Another important line of research is assessing the overall performance of an integrity system. Current integrity system literature does not have a reliable assessment design or agreement on how to measure system effectiveness (Head, Brown \& Connors 2008; Heywood \& Johnson 2017; Huberts 2014). Thus, the first step in assessing the system and the performance of integrity systems is generating valid and reliable criteria to measure performance. Research that identifies integrity risks of a NSGBs system including the coherence, coordination, capacity, instruments, structures and processes would provide important theoretical, empirical and practical contributions. In particular, examining NSGBs coordination of the integrity system at local, state/regional, and national levels and how the type of governance system influences this organisation can contribute broadly to the integrity system literature and sport literature in particular. Last, exploring what set of instruments as a whole make up an effective integrity management infrastructure is important. Research has identified a small number of instruments for ethical culture creation (i.e. ethical codes, ethical leadership, and ethics training) (e.g. Constandt et al. 2018, 2019; De Waegeneer et al. 2016). Investigating what set of integrity instruments as a whole influences integrity management effectiveness is key to understanding what ethical mechanisms limit sport integrity risks.

\section{References}

Ashby, WR 1991, Requisite variety and its implications for the control of complex systems. In: Facets of systems science. international federation for systems research international series on systems science and engineering, vol 7. Springer, Boston, MA. https://doi. org/10.1007/978-1-4899-0718-9_28

Chappelet, JL 2016, 'Autonomy and governance: necessary bedfellows in the fight against corruption in sport', in International Transparency (eds), Global corruption report: sport, Routledge, Oxon, pp. 16-28.

Chappelet, JL 2018, 'Beyond governance: the need to improve the regulation of international sport', Sport in Society, vol. 21, no. 5, pp. 724-34. doi:10.1080/17430437.2018. 1401355

Chappelet, JL, \& Mrkonjic, M 2013, 'Existing governance principles in sport: a review of published literature', in J Alm (eds), Action for good governance in international sports organisations, final report, Play the Game/Danish Institute for Sports Studies, Aarhus, pp. 222-40.

Cleret, L, McNamee, M \& Page, S 2015, “'Sports integrity” needs sports ethics (and sports philosophers and sports ethicists too)', Sport, Ethics and Philosophy, vol. 9, no. 1, pp. 1-5.

Constandt, B, De Waegeneer, E, \& Willem, A 2018, 'Coach ethical leadership in soccer clubs: an analysis of its influence on ethical behavior', Journal of Sport Management, vol. 32, no. 3, pp. 185-98.

Constandt, B, De Waegeneer, E, \& Willem, A 2019, 'Ethical code effectiveness in football clubs: a longitudinal analysis', Journal of Business Ethics, vol. 156, no. 3, pp. 621-34. 
De Waegeneer, E, Van De Sompele, J \& Willem, A 2016, 'Ethical codes in sports organisations: classification framework, content analysis, and the influence of content on code effectiveness', Journal of Business Ethics, vol. 136, no. 3, pp. 587-98.

Di Marco, A 2019, 'The internal governance of sporting organisations: international convergences on an idea of democracy', The International Sports Law Journal, vol. 19, no. 3-4, pp.171-83. doi:10.1007/s40318-019-00144-9

FIFA, 2020, Integrity support, 11 November, FIFA, Zurich.

Gardiner, S, Parry, J \& Robinson, S 2017, 'Integrity and the corruption debate in sport: where is the integrity?', European Sport Management Quarterly, vol. 17, no. 1, pp. 6-23. doi:10.1080/16184742.2016.1259246

Geeraert, A 2018, National sports governance observer. Indicators and instructions for assessing good governance in national sport federations, Play the Game/ Danish Institute for Sports Studies, Aarhus.

Geeraert, A (ed.) 2018, National sports governance observer: final report, Play the Game/ Danish Institute for Sports Studies, Aarhus.

Geeraert, A 2019, 'The limits and opportunities of self-regulation: achieving international sport federations' compliance with good governance standards', European Sport Management Quarterly, vol. 19, no. 4, pp. 520-38.

Geeraert, A, Alm, J \& Groll, M 2014, 'Good governance in international sport organisations: an analysis of the 35 Olympic sport governing bodies', International Journal of Sport Policy and Politics, vol. 6, no. 3, pp. 281-306.

Head, BW 2012, 'The contribution of integrity agencies to good governance', Policy Studies, vol. 33, no. 1, pp. 7-20. doi:10.1080/01442872.2011.601200

Head, BW, Brown, AJ \& Connors, C (eds) 2008, Promoting integrity: evaluating and improving public institutions, Ashgate (now: Taylor \& Francis), Farnham.

Heinrich, F \& Brown, AJ 2017, 'Measuring accountability performance and its relevance for anti-corruption: introducing a new integrity system-based measure', Crime, Law and Social Change, vol. 68, no. 3, pp. 359-81.

Henry, I \& Lee, PC 2004, 'Governance and ethics in sport', in J Beech \& S Chadwick (eds), The Business of Sport Management, Prentice Hall, New Jersey, pp. 25-42.

Heywood, PM \& Johnson, E 2017, 'Cultural specificity versus institutional universalism: a critique of the National Integrity System (NIS) methodology', Crime, Law and Social Change, vol. 68, no. 3, pp. 309-24.

Hoekstra, A \& Kaptein, M 2020, 'The integrity of integrity programs: toward a normative framework', Public Integrity, vol. 23, no. 2, pp. 1-13. doi:10.1080/10999922.2020.1776077

Howe, SW \& Haigh, Y 2016, 'Anti-corruption watchdog accountability: the limitations of judicial review's ability to guard the guardians', Australian Journal of Public Administration, vol. 5, no. 3, pp. 305-17. doi:10.1111/1467-8500.12185

Huberts, L 2014, The integrity of governance: what it is, what we know, what is done and where to go, Palgrave MacMillan, London. doi:10.4337/9781789900910.00040

Huberts, L \& Van Montfort, A 2020, 'Building ethical organisations: the importance of organisational integrity systems', in A Graycar (eds), Handbook on corruption, ethics and integrity in public administration, Edward Elgar Publishing, Cheltenham, pp. 449-62.

Ingle, S 2019, 'UK Athletics thrown into crisis as new CEO loses job before starting', The Guardian, 20 October.

Jayal, NG 2008, 'New directions in theorizing social accountability?', IDS Bulletin, vol. 38, no. 6, pp. 105-10. doi:10.1111/j.1759-5436.2007.tb00425.x 
Kihl, LA 2019, 'Sport integrity systems. A proposed framework'. in D Shilbury \& L Ferkins (eds), Routledge handbook of sport governance, Routledge, New York, pp. 395-409. doi: $10.4324 / 9780429440250-28$

Kihl, LA, \& Ordway, C forthcoming, 'Sport corruption', in L Wenner (eds), Oxford handbook of sport and society, Oxford University Press, Oxford.

Kinsella, L 2019, 'The 66 scandals in four years that have rocked the NRL', News.com. AU, 8 February.

Maesschalck, J \& Vanden Auweele, Y 2010, 'Integrity management in sport', Journal of Community and Health Sciences, vol. 5, no. 1, pp. 1-9.

Manoli, AE, Comille, B \& Downward, P 2020, 'Perceptions of integrity in sport: insights into people's relationship with sport', International Journal of Sport Policy and Politics, vol. 12, no. 2, pp. 1-14. doi:10.1080/19406940.2020.1747101

Molina, AD 2018, 'A systems approach to managing organisational integrity risks: lessons from the 2014 veteran's affairs waitlist scandal', The American Review of Public Administration, vol. 48, no. 8, pp. 872-85. doi:10.1177/0275074018755006

Mulgan, R 2003, Holding power to account: accountability in modern democracies, Springer, New York. doi:10.1057/9781403943835

Pedras, L, Taylor, T \& Frawley, S 2020, 'Responses to multi-level institutional complexity in a national sport federation', Sport Management Review, vol. 23, no. 3, pp. 482-97. doi:10.1016/j.smr.2019.05.001

Pope, J 2008, 'National integrity systems: the key to building sustainable, juts and honest government', in BW Head, AJ Brown, \& C Connors (eds), Promoting integrity: evaluating and improving public institutions, Ashgate (now: Taylor \& Francis), Farnham, pp. 13-31.

Sampford, C, Smith, R \& Brown, AJ 2005, 'From Greek temple to bird's nest: towards a theory of coherence and mutual accountability for national integrity systems', Australian Journal of Public Administration, vol. 64, no. 2, pp. 96-108.

Schatz, F, 2013, 'Fighting corruption with social accountability: A comparative analysis of social accountability mechanisms' potential to reduce corruption in public administration', Public Administration and Development, vol. 33, no. 3, pp. 161-74. doi:10.1002/ pad.1648

Six, F \& Lawton, A 2013, 'Towards a theory of integrity systems: a configurational approach', International Review of Administrative Sciences, vol. 79, no. 4, pp. 639-58. doi: $10.1177 / 0020852313501124$

Treviño, LK, \& Nelson, KA (eds) 2017, Managing business ethics: straight talk about how to do it right, Wiley, New Jersey.

Warren, DE, Gaspar, J \& Laufer, WS 2014, 'Is formal ethics training merely cosmetic?: a study of ethics training and ethical organisational culture', Business Ethics Quarterly, vol. 24, no, 1, pp. 85-117. 